\title{
Miller-Fisher syndrome in a patient with rheumatoid arthritis treated with adalimumab
}

\author{
Patric Thierry Kurmann • Daniel Van Linthoudt • \\ Alexander Kai-Lik So
}

Received: 29 July 2008 / Accepted: 10 September 2008 / Published online: 26 September 2008

(C) Clinical Rheumatology 2008

\begin{abstract}
Adalimumab is a frequently prescribed TNF $\alpha$ inhibitor for treatment of rheumatoid arthritis. We report on a patient who probably developed a Miller-Fisher syndrome after the second injection of adalimumab.
\end{abstract}

Keywords Adalimumab · Miller-Fisher .

Rheumatoid arthritis

\section{Introduction}

Rheumatoid arthritis (RA) is a common chronic arthropathy affecting $\approx 1 \%$ of the adult population worldwide. Tumor necrosis factor alpha (TNF $\alpha$ ) inhibitors have been increasingly used as DMARD therapy in conjunction with conventional drugs, in particular methotrexate. Adalimumab is a widely prescribed TNF $\alpha$ inhibitor. We report on a female patient with RA who developed Miller-Fisher syndrome [1] after the second injection of adalimumab.

\section{Case presentation}

The patient, a 77-year-old female with seropositive erosive RA, had previously received hydroxychloroquine, gold salts, penicillamine, sulfasalazine, and cyclosporine without

P. T. Kurmann • D. Van Linthoudt $(\bowtie)$

Department of Rheumatology, Hôpital Neuchâtelois,

CH-2300 La Chaux-de-Fonds, Switzerland

e-mail: Daniel.vanlinthoudt@ne.ch

P. T. Kurmann • D. Van Linthoudt • A. K.-L. So Department of Rheumatology and Rehabilitation, Centre Hospitalier Universitaire Vaudois,

Lausanne, Switzerland success. Methotrexate had to be discontinued because of drug-induced pneumonitis. As a result, she was maintained on azathioprine $(75 \mathrm{mg} / \mathrm{day}=1 \mathrm{mg} / \mathrm{kg})$ and prednisone $(5 \mathrm{mg} /$ day). In 2006, she underwent a cervical laminectomy C4-C5 and $\mathrm{C} 6-\mathrm{C} 7$ with plate from $\mathrm{C} 4$ to $\mathrm{C} 6$ because of a myelopathy secondary to cervical osteoarthritis. At surgery, there was no local rheumatoid pannus. Her medical history included type II diabetes mellitus, hypertension, hyperlipidemia and ischemic heart disease.

In 2007, she was started on adalimumab after a RA flare. Twenty-four hours after the second injection of $40 \mathrm{mg}$ of adalimumab, she reported dizziness and loss of cutaneous sensation that progressed proximally in the lower limbs and she was hospitalized. On admission, the general examination was unremarkable except for mild fever. The neurological examination showed ataxia of all four limbs and disappearance of the deep tendon reflexes in the lower limbs. The next day, there was a complete loss of proprioception, loss of balance and impossibility to walk. One week later, she developed a horizontal nystagmus with impossibility to lift the upper eyelids $[1,2]$.

Laboratory tests showed a raised ESR $(40 \mathrm{~mm}$ in the first hour) and CRP (69 mg/l). Autoantibodies including antiganglioside antibodies (GQ1b) were negative. The search for an infection was negative.

Radiological investigations included a cerebral and a thoraco-abdo-pelvic computed tomography, and a cervical to lumbar spine MRI; they were all normal. The electromyogram showed a decreased evocated sensitive response to peripheral nerve stimulation on the upper limbs and absence of this response on the lower limbs. The motor recording was normal. There was no muscle involvement. A diagnosis of a Miller-Fisher syndrome [1,2] was made.

Treatment consisted of high-dose steroids $(75 \mathrm{mg} /$ day $=$ $1 \mathrm{mg} / \mathrm{kg}$ ), gabapentine, and an increased dose of azathioprine $(75 \mathrm{mg}$ tad). Physical therapy was initiated. The 
biological signs of inflammation disappeared quickly but the clinical evolution was marked by a slow improvement over one year of balance, proprioception, and reflexes.

\section{Discussion}

The Miller-Fisher syndrome $[1,2]$ is an autoimmune polyneuropathy characterized by an ascending muscle weakness of the limbs, as in the Guillain-Barré syndrome, with loss of sensation, areflexia, ataxia, and opthalmoplegia. Although neurological symptoms have been reported with adalimumab [3], this case reports, to our knowledge, on the first Miller-Fisher syndrome induced by treatment with adalimumab. Immunological mechanisms have been incriminated in the development of this syndrome, though in our case, anti-ganglioside antibodies were absent. The lumbar puncture showed an isolated increased protein content $(1,000 \mathrm{mg} / \mathrm{l})$ but the patient underwent a cervical surgery a few weeks before.

Neurological complications have been described with several TNF $\alpha$ inhibitors. The use of lenercept in multiple sclerosis produced worsening of the disease causing the premature interruption of the study [4]. Similarly, 17 patients treated with etanercept [3] and two patients treated with infliximab [3] developed neurological symptoms. These latter included confusion, ataxia, dysesthesia, paresthesia, facial paralysis, optical neuritis, hemiparesia, transverse myelitis and an ascending neuropathy resembling the Guillain-Barré syndrome. All these cases were proven by a retrial of the drug. The risk was estimated for etanercept (31 cases for 100,000 patients) and infliximab (41 cases for 100,000) [3]. No case has been described with adalimumab until now. Therefore, a Miller-Fisher syndrome secondary to the use of a TNF $\alpha$ inhibitor seems to be a class effect, which can be, as in our case, rapidly and severely progressive.

Disclosures None.

\section{References}

1. Fisher M (1956) An unusual variant of acute idiopathic polyneuritis (syndrome of ophtalmoplegia ataxia and areflexia). N Eng J Med 255:57-65

2. Lo YL (2007) Clinical and immunological spectrum of the Miller Fisher syndrome. Muscle Nerve 36:915-27

3. Mohan N, Edwards ET, Cupps TR et al (2001) Demyelination occurring during anti-tumor necrosis factor alpha therapy for inflammatory arthritides. Arthritis Rheum 44:2862-2869

4. Van Oosten BW, Barkhof F, Truyen L et al (1996) Increased MRI activity and immune activation in two multiple sclerosis patients treated with the monoclonal anti-tumor necrosis factor antibody cA2. Neurology 47:1531-1534 